RUNNING HEAD: Controlling Emotional Distraction

\title{
Contrasting Reactive and Proactive Control of Emotional Distraction
}

\author{
Gina M. Grimshaw ${ }^{1}$, Laura S. Kranz ${ }^{1}$, David Carmel ${ }^{2}$, Rosie E. Moody ${ }^{1}$, \\ and Christel Devue ${ }^{1}$ \\ ${ }^{1}$ School of Psychology, Victoria University of Wellington, New Zealand \\ ${ }^{2}$ Department of Psychology, University of Edinburgh, UK
}

Address Correspondence to: Gina M. Grimshaw, School of Psychology, Victoria University of Wellington, PO Box 600, Wellington 6140, New Zealand. Gina.grimshaw@vuw.ac.nz

7972 words (excluding title page, abstract, references, tables, figures, and captions)

IN PRESS, Emotion

ACCEPTED 27 APRIL 2017 


\begin{abstract}
Attending to emotional stimuli is often beneficial, because they provide important social and environmental cues. Sometimes, however, current goals require that we ignore them. To what extent can we control emotional distraction? Here we show that the ability to ignore emotional distractions depends on the type of cognitive control that is engaged. Participants completed a simple perceptual task at fixation while irrelevant images appeared peripherally. In two experiments, we manipulated the proportion of trials in which images appeared, in order to encourage use of either reactive control (rare distractors) or proactive control (frequent distractors). Under reactive control, both negative and positive images were more distracting than neutral images, even though they were irrelevant and appeared in unattended locations. However, under proactive control, distraction by both emotional and neutral images was eliminated. Proactive control was triggered by the meaning, and not the location, of distracting images. Our findings argue against simple bottom-up or top-down explanations of emotional distraction, and instead show how the flexible use of cognitive control supports adaptive processing of emotional distractors. Keywords: Attention; Emotion; Cognitive Control; Distraction; Frequency
\end{abstract}




\section{Contrasting Reactive and Proactive Control of Emotional Distraction}

Emotional stimuli are important. They signal potential threats and rewards and so guide adaptive behaviour. Perceptual and attentional systems prioritize them, as demonstrated through behavioural, electrophysiological and neuroimaging research (for reviews see Carretié, 2014; Okon-Singer, Lichtenstein-Vidne, \& Cohen, 2013; Pourtois, Schettino, \& Vuilleumier, 2013; Yiend, 2010). But sometimes we need to ignore an emotional stimulus so we can get on with the task at hand. We might need to block out a scowling face to maintain our goal of giving a good talk, or ignore an attractive classmate to concentrate on a lecture. Emotional distractions plague us all, and in disorders such as depression, anxiety, and addiction, they can be overwhelming (Cisler \& Koster, 2010; De Raedt \& Koster, 2010; Field \& Cox, 2008). Non-emotional distractors are known to disrupt performance (Forster \& Lavie, 2008a,2008b, 2016), but they can also be controlled if we know to expect them (Braver, 2012; Müller, Geyer, Zehetleitner \& Krummenacher, 2009) Can we ever control emotional distractions as effectively as those that are more mundane?

\section{Cognitive Control of Non-emotional Distraction}

In non-emotional contexts, entirely irrelevant stimuli can disrupt performance (Forster \& Lavie, 2008a, 2008b, 2016). For example, Forster and Lavie (2008a) describe an irrelevant flanker paradigm in which participants complete a letter discrimination task near fixation, while irrelevant images (cartoon characters) appear peripherally. Even when the images are completely task-irrelevant and appear in non-target locations, they can disrupt performance (Forster \& Lavie, 2008a, 2016) as long as the participant's task is perceptually simple (i.e., low load; Forster \& Lavie, 2008b). Even under low load, distraction is not obligatory, though; distractors are also less disruptive when they appear more frequently. In 
Forster and Lavie's (2008a) experiment, images were significantly less distracting when they appeared on $50 \%$ compared to $10 \%$ of trials. Even more striking distractor frequency effects can be seen in a visual search paradigm in which a salient distractor singleton is presented on a proportion of trials. Geyer and colleagues (Geyer, Müller \& Krummenacher, 2008) report that salient singletons cause distraction when they appear on $20 \%$ or $50 \%$ of trials, but distraction is completely eliminated when they appear on $80 \%$ of trials, despite the singletons being more salient than the target (Müller et al., 2009). Finally, in a set of spatial cueing experiments, distractors in unattended locations slowed responses when they appeared rarely (on less than $20 \%$ of trials) but not when they appeared frequently (on $75 \%$ of trials; Neo \& Chua, 2006).

These frequency effects are analogous to those seen in other types of conflict tasks, such as traditional Stroop or flanker tasks, in which the distracting information shares features with the target and so can create response conflict. In such tasks, performance improves when the proportion of conflict trials increases - an effect that has been attributed to increased cognitive control when conflicts are expected (Botvinick, Braver, Barch, Carter, \& Cohen, 2001; Dishon-Berkovits \& Algom, 2000; see reviews in Bugg, 2012; Bugg \& Crump, 2012). The Dual Mechanisms of Control (DMC) model (Braver, 2012; Braver, Gray \& Burgess, 2007; Braver, Paxton, Locke, \& Barch, 2009) provides a useful framework in which to interpret the effect of distractor frequency on cognitive control. The model proposes that people fluctuate between reactive and proactive modes of control to maintain goal-relevant processing when conflicts arise. Reactive control processes are those that occur to resolve conflict after it has been detected; in contrast, proactive control optimizes control settings to prevent conflict before it occurs. Proactive strategies are more effective, but they are resource-intensive. Therefore, the model holds that reactive control 
is the default mode, but proactive control will be used when there are sufficient benefits or incentives available - for example, when frequent distractors are expected, or when rewards are available for good performance.

Although the DMC framework is often invoked to explain response-level conflict that can occur in Stroop or flanker tasks, the principles of reactive and proactive control apply across "attention, perception, and action systems" (Braver, 2012, pp. 106). In the context of an irrelevant distractor task, reactive control comprises mechanisms that occur after a distractor has been selected, such as disengagement. In contrast, proactive control mechanisms act prior to stimulus onset, altering attentional control settings to enhance the processing of targets and/or suppress the processing of distractors (see Geng, 2014 for further discussion of reactive and proactive control of distraction). A similar explanation for control of distractors is provided by Müller and colleagues, who describe the effects of distractor frequency in terms of a Dimensional Weighting Account (DWA; Müller, Heller \& Ziegler, 1995) whereby frequent distractors create an incentive to institute effortful topdown control to suppress them ${ }^{1}$.

\section{The Current Study}

We describe here two experiments that were conducted to determine whether, and how, proactive control can be used to effectively reduce or eliminate distraction from emotional images. We used the irrelevant distractor paradigm introduced by Forster and Lavie (2008a) because it has been shown to produce sizeable and robust distraction from task-irrelevant, emotionally-neutral distractors (Forster \& Lavie, 2008a, 2016), and therefore

\footnotetext{
${ }^{1}$ It is not our intention to discriminate between the DMC and DWA models of cognitive control, as both entail similar mechanisms. We focus on the DMC framework because it is a broader theory that seeks to explain cognitive control across a range of contexts, but our predictions could also be derived from the DWA.
} 
has the potential to enable comparison of emotional and neutral distraction. We thus modified the paradigm by including both emotional and non-emotional distractor images. We manipulated the frequency with which distractors appear to encourage the use of either reactive or proactive control.

Although frequent distractors are expected to engage proactive control in nonemotional contexts, it is not clear if the same should apply when distractors are emotional. The potential for emotional stimuli to attract or capture attention has been demonstrated in emotional Stroop (Phaf \& Khan, 2007), dot-probe (Mogg, Bradley, De Bono, \& Painter, 1997), visual search (Öhman, Flykt, \& Esteves, 2001), and spatial cueing tasks (Fox, Russo, \& Dutton, 2002); and their competitive advantage has been attributed to emotion-specific gain control mechanisms that are at least partly independent of classical bottom-up and top-down attentional mechanisms (Pourtois et al., 2013; Viviani, 2013). Indeed, some authors have argued that attention to emotional stimuli might be "impenetrable to cognitive control" (Öhman \& Mineka, 2001, p. 483; see also Aue, Geux, Chauvigné \& OkonSinger 2013; Aue, Chauvigné, Bristle, Okon-Singer, \& Geux, 2016), meaning that top-down processes might fail to regulate responses to emotional distractors. Pessoa (2009) has further argued that the processing of high arousal emotional stimuli draws on "common pool resources" - the same resources that are recruited for executive control - and can therefore disrupt the recruitment of control processes. If so, increasing distractor frequency may not engender the same shift to more effective proactive control for emotional stimuli as it does for neutral ones.

A further question, which we address in both experiments, concerns the effects of valence on emotional distraction. Although research on emotional attention is dominated 
by the study of negative (specifically threat-related) stimuli, positive and negative images are equally engaging when equated for arousal and biological relevance (e.g., Most, Smith, Cooter, Levy, \& Zald, 2007; Vogt, De Houwer, Koster, Van Damme, \& Crombez, 2008). We therefore expected they would be equally distracting when distractors are rare. However, positive and negative distraction might not be equally well controlled when distractors are frequent. Proactive control is subject to motivational factors (Botvinick \& Braver, 2015; Chiew \& Braver, 2011), and because positive images are inherently rewarding, participants might be less motivated (or less able) to control the distraction they cause than they are with negative images (Gupta et al., 2015; Pearson, Donkin, Tran, Most, \& Le Pelly, 2015). If so, then positive images would be expected to cause more distraction than negative images under high distractor frequency.

\section{EXPERIMENT 1}

The goals of Experiment 1 were twofold. Our primary goal was to determine the effect of distractor frequency (a manipulation of cognitive control) on both emotional (positive and negative) and non-emotional distraction. To that end, half our participants were presented with distractor images on $25 \%$ of trials (encouraging reactive control), and half were presented with distractor images on $75 \%$ of trials (encouraging proactive control).

We additionally used this experiment to ensure that emotional images were more distracting than neutral images, at least when they appeared infrequently (that is, under reactive control conditions). Although emotional stimuli are commonly claimed to demand attention, some studies have shown that they do not do so when they are task-irrelevant and appear in non-target locations (Lichtenstein-Vidne, Henik, \& Safadi, 2007; Okon-Singer, Tzelgov, \& Henik, 2007; Vromen, Lipp, \& Remington, 2015). Given that we are concerned 
with the effect of cognitive control manipulations on emotional distraction, it is important that such distraction be observed.

\section{Method}

\section{Participants}

Participants in both experiments were fluent English speakers with normal or corrected vision. They received course credits or movie vouchers in exchange for their participation. The Human Ethics Committee at Victoria University of Wellington approved the experiments.

As these are the first experiments to use emotional pictures with the peripheral distractor paradigm (see Stimuli and Procedure, below), we had no prior effect size to use in a power analysis. We therefore used data from a pilot experiment $(\mathrm{N}=76$; none of whom participated in the other experiments reported here) in which distractors were present on $50 \%$ of trials to estimate the sample size required to detect emotional distraction (that is, greater distraction from emotional than from neutral images). The effect size of emotional distraction in the pilot experiment $\left(d_{z}=0.38\right)$ indicated 44 participants per condition to achieve power of .80 to observe an emotional distraction effect. For counterbalancing purposes, we ran 96 participants (64 female; mean age 19.08 years, $S D=1.70$ ) who were randomly assigned to either the low distractor frequency $(\mathrm{N}=48)$ or high distractor frequency $(\mathrm{N}=48)$ condition.

\section{Stimuli and Procedure}

Participants sat in a dimly-lit room, in separate cubicles (up to four; participants could not see each other or any screen but their own), viewing 24" AOC monitors (refresh 
rate $120 \mathrm{~Hz}$, resolution of $1920 \times 1080$ pixels) from a distance of $57 \mathrm{~cm}$. Head position was stabilized by a chin-rest. Stimulus presentation and response collection were controlled by Dell Precision T1700 computers, using E-prime 2.0 software (Psychology Software Tools, Pittsburgh PA).

Visual stimuli were presented on a black background. Each trial began with a white fixation cross at the center of the screen (random duration between 416 and $833 \mathrm{~ms}$ ), which was replaced by the 100 ms task display consisting of six white letters arranged in a circle around fixation (radius $1.75^{\circ}$ ). A target letter (" $\mathrm{X}$ " or " $\mathrm{N}$ "; height $0.67^{\circ}$, width $0.36^{\circ}$ ) appeared in one location; small "o"s $\left(0.22^{\circ} \times 0.22^{\circ}\right)$ occupied the other five locations. On a proportion of trials ( $25 \%$ or $75 \%$, depending on condition), a peripheral distractor image $\left(6.68^{\circ} \times 6.68^{\circ}\right.$, centered at $6.68^{\circ}$ eccentricity) was presented simultaneously above or below the letter display. Distractors were 12 negative (mutilations), 12 positive (erotic couples), and 12 neutral (always depicting people) images selected from the International Affective Picture System (IAPS; Lang, Bradley, \& Cuthbert, 2008) (Figure 1). Positive and negative images were chosen to be similarly high in arousal. Because there are sex differences in ratings of erotic and mutilation images (Lang et al., 2008), stimulus sets differed slightly for male and female participants to ensure similar valence and arousal ratings overall (Table 1). Images were matched for luminance and contrast using the Shine image toolbox in Matlab (Willenbockel et al., 2010).

Participants responded during a 1900 ms window following stimulus offset, using a key press to indicate which letter had been presented. Participants used index and middle fingers of their right hand to press " 1 " and " 2 " (for " $X$ " and " $N$ ", respectively) on the keyboard's number pad. They were instructed to respond as quickly as possible without 
sacrificing accuracy, and to ignore any peripheral images. The 1900 ms response window elapsed regardless of whether a response was made; participants then received visual feedback for $100 \mathrm{~ms}$ ("correct" or "incorrect", as appropriate). A variable inter-trial interval (ITI), equal to the trial's initial fixation cross duration, preceded the next trial.

Participants completed 8 blocks of 48 trials, each consisting of four repetitions of the 12 possible combinations of two target-letters (i.e. " $\mathrm{X}$ " or " $\mathrm{N}$ ") and six target locations within the letter array. On distractor-present trials, IAPS images were randomly presented above or below the display, with the nearest edge appearing $3.34^{\circ}$ from fixation. Within a block, all images were of the same valence. In the $25 \%$ condition each of the 12 distractor images was presented once in each block; in the $75 \%$ condition each distractor image was presented three times in each block. Trials within a block were randomized with the constraint that all 12 images were presented before they repeated. The eight blocks were separated into two super-blocks that were separated by a 5-minute filler task (Sudoku). In one super-block, the sub-blocks were negative and neutral (presented in ABBA order), and in the other the sub-blocks were positive and neutral (presented in the same ABBA order). Order of super-blocks, and of sub-blocks within super-blocks, were counterbalanced across participants. Participants completed 12 practice trials without distractors before the experimental blocks, to familiarize themselves with the task. To encourage participants to adopt the intended control strategy (reactive or proactive), instructions provided at the beginning of the experimental blocks informed them of the frequency with which distractors would appear.

After the experimental task, participants completed the Attentional Control Scale (Derryberry \& Reed, 2002), the Mini Mood and Anxiety Symptom Questionnaire (Casillas \& 
Clark, 2000), and the Kinsey Scale of Sexual Orientation (Kinsey, Pomeroy, \& Martin, 1948).

These were included as exploratory measures to guide future research, and were not considered in analyses.

\section{Data Analysis}

Analyses were undertaken only after all data were collected. Each participant's mean response time (RT) in each condition was calculated for correct trials only. Response times less than $200 \mathrm{~ms}$ from stimulus onset were excluded as anticipatory responses. The primary dependent variable used in analyses was the distraction index (calculated in each block as mean RT for distractor-present trials minus mean RT for distractor-absent trials). Reported analyses focus on distraction indices as these are most relevant to hypotheses; however, for completeness, mean accuracies, response times, distraction indices, effect sizes, and confidence intervals for all conditions are reported in tables. Reported effect sizes for paired comparisons were adjusted for the correlation between measures, yielding effect sizes $\left(d_{r m}\right)$ that are comparable to those produced in independent study designs (Cohen's $d_{s}$; Dunlap, Cortina, Vaslow \& Burke, 1996). Effect sizes based on within-subject differences scores $\left(d_{z}\right)$ are used for sample size calculations. Degrees of freedom were adjusted for heterogeneity of variance and for violations of sphericity (Greenhouse-Geisser) where necessary. Error bars in all figures are 95\% confidence intervals, adjusted for within-subject comparisons (Morey, 2008) where appropriate.

\section{Results and Discussion}

Distraction depended critically on both distractor frequency and emotionality (see Figure $\mathbf{2}$ and Table 2). As predicted by the DMC model, infrequent images caused more distraction than frequent ones; this was true for emotional as well as neutral images. 
Furthermore, although emotional images were more distracting than neutral ones in both low and high frequency conditions, this difference was substantially attenuated when distractors were frequent.

These conclusions are supported statistically by Analysis of Variance (ANOVA) on distraction indices. Distraction in neutral blocks did not differ across the negative and positive halves of the experiment, $t(95)=.27, p>.250, d_{r m}=0.033$, so neutral blocks were combined to create one distraction index for neutral trials. Distraction indices were then analysed in a 2-way mixed ANOVA with distractor frequency (low vs. high) as a betweensubjects factor, and valence (negative, neutral, positive) as a within-subjects factor. A main effect of frequency showed that overall distraction was markedly attenuated in the high frequency condition, $F(1,94)=25.73, p<.001, \eta_{p}{ }^{2}=.215$, consistent with the engagement of proactive control when distractors were expected to appear frequently. There was also a main effect of valence, $F(2,188)=14.82, p<.001, \eta_{p}{ }^{2}=.136$, indicating greater distraction by emotional than neutral images. Importantly, frequency interacted with valence, $F(2,188)$ $=3.93, p=.026, \eta_{p}{ }^{2}=.040$, and in a post-hoc follow-up analysis with the quadratic effect of valence, $F(1,94)=7.37, p=.008, \eta_{p}^{2}=.040^{2}$. In the low frequency condition, neutral images were less distracting than both negative, $t(47)=3.71, p=.001, d_{r m}=0.532$, and positive images, $t(47)=4.37, p<.001, d_{r m}=0.663$, which did not differ, $t(47)=.10, p>.250, d_{r m}=$ 0.012. In the high frequency condition, neutral images were also less distracting than both negative, $t(47)=2.36, p=.022, d_{r m}=0.501$, and positive images, $t(47)=2.35, p=.023, d_{r m}=$ 0.205 , which did not differ, $t(47)=.18, p>.250, d_{r m}=0.036$. Crucially, the interaction was driven by the fact that the difference between emotional and neutral distraction was

\footnotetext{
${ }^{2}$ Because we entered neutral images as the middle level of the valence variable, a quadratic effect was expected if negative and positive images were more distracting than neutrals, but did not differ from each other.
} 
significantly attenuated in the high compared to the low frequency condition for both negative (15 ms vs. $44 \mathrm{~ms}), t(71.407)=2.17, p=.033, d_{s}=0.443$, and positive images (14 ms vs. $45 \mathrm{~ms}), t(74.389)=2.64, p=.010, d_{s}=0.539$

Accuracy was very high overall $(M=96.22 \%, S D=2.39)$ and did not differ between conditions (see Table 3). Distraction indices for accuracy were analysed in a 2-way ANOVA similar to that used for RT distraction indices, with distractor frequency (low vs. high) as a between-subjects variable, and valence (negative, neutral, positive) as a within-subjects variable. No main effects or interactions were observed, all $p s>.20$, ruling out explanation of the RT-based effects in terms of speed-accuracy tradeoffs.

As predicted by the DMC framework, distraction from frequent images was much lower than from infrequent ones. This finding is consistent with the successful engagement of proactive control in the high-frequency condition. An alternative explanation must be considered, though: Repeated exposure to images in the high frequency condition may have reduced the images' distraction potency through habituation. Across the experiment, each image was presented six times in the high frequency condition, but only twice in the low frequency condition. If habituation accounts for attenuated distraction in the high frequency condition, then attenuation should not be apparent at the beginning of the experiment, before habituation could occur.

To test this alternative account, distraction indices were calculated based only on the first 12 distractor-present and distractor-absent trials of the experiment (for each participant, these were either positive, negative, or neutral distractor trials, and so image type becomes a between-subjects variable in this analysis). Note that images were never repeated across these trials. Because there were no effects of valence in either frequency 
condition ( $p^{\prime} s>.70$ for the comparison of positive and negative distraction indices), we maximised power by collapsing positive and negative conditions here to create an "emotional" image category. Distraction indices were then analysed in a 2 (emotional vs. neutral distractors) $\times 2$ (low vs. high distractor frequency) between-subjects ANOVA. There was a main effect of emotionality, $F(1,92)=4.44, p=.04, \eta_{p}{ }^{2}=.046$, reflecting greater distraction from emotional than from neutral images. Although the interaction with distractor frequency fell short of significance, $F(1,92)=2.55, p=.11, \eta_{p}{ }^{2}=.027$, (likely due to reduced power inherent in the use of only a small subset of trials and a between-subjects manipulation of emotionality), examination of Figure 3 clearly shows that the effect of emotionality is driven by the low frequency condition. Specifically, the pattern here is consistent with that seen over all trials: emotional images were more distracting than neutral images under low distractor frequency, $t(30.76)=2.20, p=.04, d_{s}=0.449$, but not under high distractor frequency, $t(46)=0.48, p>.250, d_{s}=0.139$. This attenuation in the high frequency condition is not consistent with a habituation account, which would predict similar distraction in low and high distractor frequency conditions at the beginning of the first experimental block. High distractor frequency thus leads to good control of emotional images, even before any image repetition has occurred. This rapid adoption of a proactive strategy may have been facilitated by the pre-experiment instructions we provided to participants to expect distractors to appear either frequently or rarely.

To determine whether there might still be a differential reduction in distraction over the course of the experiment that could account for the effects of distractor frequency, we repeated the analysis but included the first 12 distractor present and absent trials of Block 1 , and the last 12 distractor present and absent trials of Block 4 (i.e., the first and last blocks of 
a super-block; as Block 4 always had the same type of distractor image as Block 1). The main effects of distractor frequency, $F(1,92)=10.740, p=.001, \eta_{p}^{2}=.105$, emotionality, $F(1,92)=$ 7.201, $p=.009, \eta_{p}{ }^{2}=.073$, and their interaction, $F(1,92)=3.958, p=.050, \eta_{p}{ }^{2}=.041$ parallel the effects in the main experiment. There was a marginal effect of block, $F(1,92)=2.997, p$ $=.087, \eta_{p}{ }^{2}=.032$, reflecting a modest decrease in distraction over the course of the first super-block. Importantly, this effect of block did not interact with any other effects (all $p$ 's > .352), meaning that it cannot account for the effects of distractor frequency on either emotional or neutral distraction.

In summary, performance in the low frequency condition shows that emotional images can be more distracting than non-emotional ones, even if they are task-irrelevant and appear in unattended locations. But the high frequency condition shows that distraction from both emotional and neutral images can be well controlled when they are expected to appear frequently. Although there was still a small emotional distraction effect (that is, significantly greater distraction from emotional than neutral images) in the high frequency condition, it was markedly attenuated compared to that observed in the low frequency condition. The effect of distractor frequency is consistent with that seen in other (nonemotional) conflict tasks such as Stroop or flanker paradigms, in which increasing proportions of conflict trials are associated with reduced interference as a function of proactive control (Bugg \& Crump, 2012), and in visual search experiments in which salient singletons are distracting when they appear rarely, but can be suppressed when they appear frequently (Müller et al., 2009). Our findings suggest that the DMC framework can be extended to predict distraction in emotional contexts. In Experiment 2, we further 
investigate the nature of the proactive control that is applied when frequent distractors are expected.

\section{EXPERIMENT 2}

Proactive control is not a mechanism in and of itself; it is a collective term for a set of mechanisms that optimize performance when challenges are anticipated or there are incentives for control (Braver, 2012). What specific mechanism(s) support the effective control of emotional and neutral distraction when distractors appear frequently?

One possibility is that proactive control is location-based: when distractors are frequent, participants may alter attentional settings to enhance visual processing at target locations and inhibit processing at distractor locations (Foxe \& Snyder, 2011; Geng, 2014). By this logic, high-frequency distractors - both neutral and emotional - lose potency in the high frequency condition because their potential locations receive insufficient processing to trigger attentional capture. Importantly, this location-based hypothesis suggests that proactive control mechanisms should not be sensitive to the meaning of irrelevant images, just to their presence. Alternatively, activation of proactive control may be based on meaning rather than location: the resources for distractor suppression may be recruited only if meaningful images are expected to appear frequently.

To distinguish between these alternatives we repeated the high and low frequency conditions of Experiment 1 but included a third condition, in which distracting images were also presented on $75 \%$ of trials; however, of those, one third ( $25 \%$ of trials) were intact images, and two thirds ( $50 \%$ of all trials) were pixelated scrambles of the same images. Therefore, although the overall frequency of distractors in this combined condition was equivalent to that in the high frequency condition, only $25 \%$ of trials featured an intact (i.e., 
meaningful) distractor, as in the low-frequency condition. If proactive control is locationbased and driven by the anticipation of any distractor, then distraction in the combined condition (for both intact and scrambled images) should be well controlled - just like it was in the high frequency condition in Experiment 1. However, if proactive control mechanisms are recruited only when meaningful distractors are expected to appear frequently, then distraction caused by the infrequent intact images in the combined condition should more closely resemble that caused by meaningful images in the low frequency condition.

\section{Method}

\section{Participants}

123 participants ( 85 females; mean age $=19.12, S D=2.49$ ) were randomly assigned to one of three possible conditions: low distractor frequency $(N=40)$, high distractor frequency $(N=41)$ or combined distractors $(N=42)$. One participant who did not follow instructions was replaced with another in the same condition, and is not included in the final participant count or demographics, nor in any analyses. Sample size calculations were based on the critical comparison between the difference in emotional compared to neutral distraction in the low frequency $(M=45 \mathrm{~ms}, S D=68 \mathrm{~ms})$ and high frequency $(M=14 \mathrm{~ms}, S D$ $=37 \mathrm{~ms}$ ) conditions from Experiment 1 (capturing the frequency $\mathrm{x}$ valence interaction). The effect size $\left(d_{s}=0.56\right)$ led to an estimated sample of 40 participants per condition to achieve power of .80 .

\section{Stimuli and Procedure}

Pixel-scrambled versions of the IAPS images used in Experiment 1 were created by dividing each image into 1296 squares and rearranging them randomly. Scrambling removed 
all recognizable elements while keeping overall color and luminance constant. Stimuli and task were otherwise identical to those in Experiment 1, except that target letters were " $\mathrm{K}$ " (replacing " $\mathrm{X}$ ") or “ $\mathrm{N}$ ".

Participants completed three counterbalanced blocks of 96 trials, with a different image valence (positive, neutral, or negative) in each block. Distractors appeared on $25 \%$ of trials in the low frequency condition and on $75 \%$ of trials in the high frequency condition. In the combined condition, distractors appeared on $75 \%$ of trials, but $1 / 3$ of these were intact images ( $25 \%$ of trials) and $2 / 3$ were scrambled images of the same valence ( $50 \%$ of trials). As in Experiment 1, trials were presented in random order within a block, with the constraint that images (intact and scrambled) were not repeated until they had all been presented.

Participants were again instructed to expect distractors on either $25 \%$ or $75 \%$ of trials. Prior to the experimental blocks, they completed 24 practice trials on which scrambled distractors were presented at the same distractor proportion that they would experience in the experimental blocks. This experience with the appropriate distractor frequency during the practice trials was intended to further encourage use of either a reactive or proactive strategy from the outset of the experiment.

Data were analysed as in Experiment 1.

\section{Results and Discussion}

Despite minor changes in design and blocking procedure, results replicated those of Experiment 1 for the low and high distractor frequency conditions: infrequent images were overall more distracting than frequent ones, and emotional images were more distracting than neutral images in the low distractor frequency condition only. Importantly, in the 
combined condition, intact images produced similar distraction to those in the lowfrequency condition, a pattern that is consistent with meaning-based but not with locationbased deployment of proactive control (Figure 4 and Table 4).

The factor of distractor type (intact or scrambled) was manipulated only in the combined condition, so the full experimental design could not be analysed in a single ANOVA. Rather, we ran four analyses that were planned a priori, each targeting a different question. The first compared distraction in the low and high distractor frequency conditions, to verify that we had replicated Experiment 1; the second and third analyses compared distraction from intact images in the combined condition to that in the low and high frequency conditions, respectively, in order to determine whether it differed significantly from either of them. The fourth analysis compared distraction from intact and scrambled images within the combined condition, to determine whether control differed for meaningful and meaningless distractors.

In the first analysis, comparison of the high and low frequency conditions revealed the same main effects of frequency, $F(1,79)=13.57, p<.001, \eta_{p}{ }^{2}=.147$, and valence, $F(2$, 158) $=3.49, p=.033, \eta_{p}^{2}=.042$, as were observed in Experiment 1 . Although the interaction between distractor frequency and valence was not significant, $F(2,158)=2.23, p=.135, \eta_{p}^{2}$ $=.025$, the quadratic interaction was, $F(1,79)=3.99, p=.049, \eta_{p}{ }^{2}=.048$. In the low frequency condition, neutral images were less distracting than both negative, $t(39)=2.97, p$ $=.005, d_{r m}=0.513$, and positive images, $t(39)=2.29, p=.028, d_{r m}=0.379$, which did not differ, $t(39)=.24, p>.250, d_{r m}=0.029$. In the high frequency condition, there was no distraction from either emotional or neutral images (see distraction indices in Table 4). 
Because the high and low frequency conditions here are close replications of the high and low frequency conditions in Experiment 1 (with only minor changes in block structure), we combined effect sizes for distraction indices in a mini meta-analysis. Across experiments, the low frequency conditions produced significant distraction for negative, $d=$ $0.416,95 \% \mathrm{Cl}[.201, .630]$, positive, $d=0.354,95 \% \mathrm{Cl}[.142, .566]$, and neutral images, $d=$ $0.219,95 \% \mathrm{Cl}[.011, .427]$. In the high frequency conditions, no distraction was observed for any image type (negative images, $d=.122,95 \% \mathrm{Cl}[-.085, .328]$; positive images, $d=0.106$, $95 \% \mathrm{Cl}[-.098, .311]$; neutral images, $d=0.050,95 \% \mathrm{Cl}[-.154,254])$. While emotional images were slightly more distracting than neutral images in Experiment 1, the meta-analysis shows that emotional distraction in the high frequency condition is not robust. The meta-analysis strengthens our conclusion that emotional images are more distracting than neutrals under conditions that promote reactive control (i.e. low distractor frequency), but also that both emotional and neutral images can be effectively controlled when expectations bias control toward proactive mechanisms.

The second comparison, of the intact-combined and low frequency conditions, showed only a main effect of valence, $F(2,160)=4.56, p=.012, \eta_{p}{ }^{2}=.054$. Neutral images were less distracting than both negative images, $t(81)=2.55, p=.013, d_{r m}=0.340$, and positive images, $t(81)=2.60, p=.011, d_{r m}=0.322$, which did not differ, $t(81)=.08, p>.250$, $d_{\mathrm{rm}}=0.009$. Importantly, condition did not interact with valence, $F(2,160)=.60, p>.250, \eta_{p}^{2}$ $=.007$, nor with the quadratic effect of valence, $F(1,80)=1.12, p>.250, \eta_{p}{ }^{2}=.014$, meaning that the pattern of greater distraction from emotional than neutral images did not differ between the two conditions. 
In contrast, our third comparison of the intact-combined and high frequency conditions revealed only a main effect of condition, $F(1,81)=6.60, p=.012, \eta_{p}^{2}=.075$, reflecting greater distraction from intact images in the combined than in the high frequency condition. Distraction from intact images in the combined condition therefore more closely resembles that of the low frequency than of the high frequency condition.

Our final analysis focused on the combined condition alone, to compare distraction caused by intact and scrambled images. If proactive control is directed to specific locations (but not content), then we expect both intact and scrambled images to be similarly controlled. However, as can be seen on the right side of Figure 4, even within the same block of trials, intact images caused distraction but scrambled versions of the same images did not. A 3 (Valence: negative, neutral, positive) x 2 (Distractor Type: intact, scrambled) repeated measures ANOVA on distraction indices showed only a main effect of distractor type, confirming that intact images were significantly more distracting than scrambles, $F(1$, $41)=26.04, p<.001, \eta_{p}^{2}=.388$. Intact images of all valences produced significant distraction, but scrambles did not (Table 4).

The excellent control of scrambles - in the context of distraction by intact images presented in the same block - is somewhat surprising. In combined blocks, participants could not anticipate what type of image would appear. It is clear that they adopted a control strategy, as scrambles produced no distraction whatsoever. However, that strategy appears to be flexible enough to eliminate distraction from irrelevant scrambles while still allowing disruption by infrequent intact images. We return to this issue in the General Discussion. Because the scrambles were constructed from the intact images, their control also argues 
against an explanation of emotional distraction in terms of low level visual features such as colour or luminance.

Accuracy was very high overall $(M=96.51 \%, S D=2.63$; see Table 5). Distraction indices for intact image accuracies were analysed in a 2-way ANOVA with valence (negative, neutral, positive) as a within-subjects variable, and distractor frequency (low, high, combined) as a between-subjects variable. We observed a main effect of valence, $F(2,240)$ $=7.54, p=.001, \eta_{p}{ }^{2}=.059$ that did not interact with distractor frequency, $F(4,240)=1.58, p$ $=.180, \eta_{p}{ }^{2}=.026$. This small effect was in the opposite direction to that which would suggest a speed-accuracy trade-off, and is consistent with that seen in RTs; that is, less distraction from neutral $\left(M=-1.22 \%, S D=4.76, d_{r m}=0.25\right)$ than from positive $(M=0.37 \%$, $S D=4.41, d=0.08)$ or negative images $\left(M=0.85 \%, S D=4.58, d_{r m}=.019\right)$, which did not differ.

\section{GENERAL DISCUSSION}

Emotional distractors can be more potent than neutral ones. But we are not slaves to distraction; when we expect them to appear frequently, both emotional and neutral distractors can be effectively controlled. Our findings suggest that reactive and proactive control mechanisms deal with emotional distractions differently.

We used a distractor frequency manipulation to encourage either a reactive control strategy (when distractors were rare) or a proactive control strategy (when distractors were frequent). In other conflict paradigms such as the Stroop task, a high proportion of conflict (i.e., incongruent) trials induces a proactive control strategy (Bugg, 2012; Bugg \& Crump, 2012; Gonthier, Braver, \& Bugg, 2016), and similar effects of distractor frequency are observed in spatial attention tasks (e.g., Geyer et al., 2008). In both experiments reported 
here, infrequent emotional images - both positive and negative - were more distracting than non-emotional ones, despite being entirely irrelevant to the task and appearing in unattended locations. We therefore show clear evidence that irrelevant emotional distractors can capture attention, at least under experimental conditions in which few of them are expected. However, both emotional and neutral distraction were effectively eliminated (as indicated by the meta-analysis) when irrelevant images appeared frequently, suggesting that proactive mechanisms are effective at controlling both emotional and nonemotional distraction. Positive images were controlled as effectively as negative ones, even though we might expect participants to be less motivated to ignore them (Botvinick \& Braver, 2015; Gupta et al., 2015). Our findings show that the predictions of the DMC framework can be extended to emotional contexts.

Task-irrelevant emotional information therefore appears to be prioritized under reactive control, but not under proactive control. We speculate that these two modes of control allow for flexibility in the response to emotional distractors. When they appear rarely, emotional distractors may carry important information that should override ongoing processing. It is therefore adaptive for distraction in reactive contexts to be stimulus-driven. However, when distractors - even emotional ones - appear frequently, and without consequence, their informational value is limited. In these contexts, proactive mechanisms allow for control of both emotional and non-emotional distraction and so facilitate goalrelevant processing.

A dynamic shift in the control of emotional distraction may well account for some discrepancies that have appeared in the literature regarding the ability of emotional images to capture attention, where some studies find that irrelevant emotional images that occur 
outside the focus of attention can disrupt processing (Fox, Dutton, Yates, Georgiou, \& Mouchlianitis, 2015; Junhong et al., 2013; Yates, Ashwin, \& Fox, 2010), but others show they do not (Lichtenstein-Vidne et al., 2012; Okon-Singer et al., 2007; Vromen et al., 2015). A systematic manipulation of proportion conflict may furthermore shed light on the modulation of other emotional effects such as those revealed with emotional Stroop, visual search, or spatial cueing paradigms. Task demands may determine the extent to which reactive or proactive control mechanisms are engaged, and consequently whether or not emotional distraction is observed. Although we manipulated distractor frequency to alter control strategy in the present experiments, other factors like difficulty, feedback, testing environment, or incentives for performance might be equally important in determining control. For example, a recent study in our lab (Maddock, Harper, Carmel \& Grimshaw, submitted) shows that financial rewards (which are thought to encourage use of proactive control; Botvinick \& Braver, 2015) can also attenuate emotional distraction. One advantage of interpreting emotional distraction effects within the DMC framework (Braver, 2012) is that it makes clear predictions about when emotional distraction should (and should not) be observed.

Although we believe these to be the first studies to use the frequency of emotional distractors to manipulate cognitive control, several recent studies using different paradigms point to an important role for control processes in determining the extent of emotional distraction. Some evidence comes from a study by Augst and colleagues (Augst, Kleinsorge \& Kunde, 2014, Experiment 4), who examined the effect of a central distractor image on ability to compare flanking targets in a line-orientation matching task. Although distractors were present on every trial, they manipulated the proportion of emotional and neutral distractors within a block such that the dominant image type was present on $80 \%$ of trials. 
An interaction of distractor valence and context was broadly consistent with our findings here - images were more distracting when they were of the rare valence than the dominant valence. However, in contrast to our finding that distractor frequency has clear effects on control of emotional images, their effects were driven predominantly by the effect of context on performance when distractors were neutral, not emotional. Specifically, response times to trials with negative or positive distractors were the same regardless of their proportion within a block, but response times on trials including neutral distractors were greater when they were rare than when they were frequent. At present there are so few studies on control of emotional distraction that it is premature to speculate on the causes of variability across studies, and further studies using different paradigms will be important for establishing how experimental parameters affect control.

Shifting to temporal attention, Most and colleagues (Most, Chun, Widders \& Zald, 2005) used a variant of the attentional blink paradigm to show that threatening images interrupt processing of a subsequent target image - an effect called emotion-induced blindness. However, some participants (those low in harm avoidance) were able to use foreknowledge of the target identity to reduce emotional distraction. Most and colleagues argue that knowledge about the target allowed those participants to adopt a specific attentional set - that is, a proactive control strategy - that enhanced target processing and attenuated distractor processing.

Our findings are therefore consistent with others suggesting that proactive control can be used to reduce the impact of emotional distractors when they are irrelevant to our goals. However, they stand in contrast to findings reported by Aue and colleagues (Aue et al., 2013, 2016), who show that proactive control does not facilitate attention to emotional 
stimuli when they are goal-relevant. Their participants performed a visual search task in which a discrepant target was either a spider or a bird. Pre-cueing the identity of the target (a manipulation that should trigger proactive control to guide search) facilitated detection of target birds, but had no effect on detection of target spiders. It is possible that under conditions in which emotional stimuli are goal-relevant their processing is already prioritized (Lichtenstein-Vidne, Henik, \& Safadi, 2012; Vromen, Lipp, \& Remington, 2015), making further manipulations of control unnecessary. We note that proactive control is not a unitary mechanism, and the type of control recruited to aid search for a target maybe be very different from that recruited to suppress a distractor. Further comparisons across experimental paradigms and task parameters will be useful for testing the boundary conditions of cognitive control in emotional contexts.

How might higher distractor frequency facilitate proactive control? The combined condition in Experiment 2 provides some clues. Here we tested whether effective control of distractors in the high frequency condition was based on inhibition of potential distractor locations (Foxe \& Snyder, 2011; Geng, 2014). If so, both scrambled and intact images should have been effectively controlled in the combined condition. Instead, intact images produced distraction equivalent to that in the low frequency condition, but scrambled images produced no distraction at all. The effective control of both emotional and neutral images in the high frequency condition therefore depends on the frequent presentation of meaningful content.

Although these findings allow us to rule out location-based suppression as the mechanism by which proactive control reduces distraction, several non-exclusive alternatives remain. One possibility is that proactive control enables rapid disengagement 
from distractors (Theeuwes, 2010; see also Geng, 2014). We think this is unlikely to be the case in our experiments though, as both high frequency intact images and scrambles in Experiment 2 produced no observable distraction, suggesting that they did not engage attention at all. An alternative distractor-based explanation is that proactive mechanisms might allow participants to rapidly (perhaps pre-attentively) categorize distractors as meaningless scrambles or intact meaningful images (Li, VanRullen, Koch \& Perona, 2002; Peelen, Li \& Kastner, 2009), and then either suppress them or select them for further processing. According to this view, images could be suppressed if they were meaningless scrambles (in the combined condition), or if they were intact images that occurred frequently (in the high frequency condition), but could be selected for further processing in the case of an infrequent intact image (in either the low frequency or combined condition). Such a mechanism is consistent with the signal suppression hypothesis (Gaspelin, Leonard \& Luck, 2015, 2017; Sawaki \& Luck, 2010) which holds that salient stimuli generate an obligatory "attend-to-me" signal, which can be suppressed before an attentional shift occurs if such a shift would be contrary to top-down goals. Although the signal suppression hypothesis has been tested in the context of salient color singletons, emotional stimuli are also salient stimuli that might be subject to similar top-down control under some circumstances.

Finally, proactive control might act by enhancing the processing of targets (Egner \& Hirsch, 2005) so that they compete more effectively against distractors (Desimone \& Duncan, 1995). Such enhancement could be graded according to expectations about distractors. For example, in the high frequency condition in which distractors are expected to appear often and have limited informational value, target enhancement might be maximal, leading to minimal processing of distractors. In the combined condition, in which 
intact distractors are potentially important (because they are rare), enhancement might be graded so that intact images, but not scrambles, are able to compete with targets. It is difficult to distinguish between distractor-based and target-based mechanisms on the basis of behavioural data alone, and so electrophysiological methods that are able to separate their effects (Gaspar \& McDonald, 2014; Hickey, Di Lollo, \& McDonald, 2009; Hilimire, Mounts, Parks, \& Corballis, 2011) will be valuable in identifying the specific mechanisms that produce effective control.

Although the exact mechanisms underlying proactive control over irrelevant distractors remain to be elucidated, we show here that they can operate effectively over both neutral and emotional images. A key aspect of our experimental design is that distractors were blocked by valence. Although participants did not know exactly when or where a distractor might appear, they could predict its emotional value. We used this blocking procedure to maximise any potential emotional distraction, as stronger emotion effects are sometimes observed with blocked than with mixed presentation (e.g., in emotional Stroop tasks; Williams, Matthews, \& MacLeod, 1996, and in visual search with emotional distractors; Devue, Bepolsky, \& Theeuwes, 2011). Our findings therefore require replication using a mixed presentation format, to determine whether similarly effective control is possible when participants cannot predict the nature of an upcoming distractor.

Our blocking procedure does raise the interesting possibility that participants tailored their cognitive control to the type of distractor they expected. Specifically, blocks with emotional distractors may have required more effortful control than blocks with neutral distractors. Psychophysiological measures that can provide direct evidence of proactive control, such as pre-trial EEG alpha suppression (Mazaheri, DiQuattro, Bengson, \& 
Geng, 2011) or pupil dilation (Chiew \& Braver, 2013), will be useful in further investigations probing control mechanisms to determine whether they are emotion-specific.

Another factor in our design is that expectations about upcoming distractors were manipulated by controlling the frequency with which they appeared. In other conflict paradigms (e.g., Stroop, flanker tasks), increasing the proportion of conflict trials is thought to engage voluntary and global proactive control mechanisms that are sustained across trials (for a review, see Bugg \& Crump, 2012). However, control might also be triggered more locally by the presence of a distractor on a preceding trial through a mechanism of conflict adaptation (Botvinick, Braver, Barch, Carter, \& Cohen, 2001), which of course occurs more often in the high frequency condition. The experiments we report here were not designed to probe sequential trial effects, which could be used in future work to determine the relative contributions of both types of control.

Although high frequency distractors led to better control overall, the confidence intervals show that people varied considerably in their level of distraction under both reactive and proactive conditions. The DMC framework suggests that the use of proactive versus reactive control is not only determined by task or situational factors, but also by individual or group-level differences in the use of control. Variability might therefore reflect individual differences in ability to control distraction generally (Forster \& Lavie, 2016). Alternatively, it might reflect differences in how effectively people deploy proactive control (Braver, 2012), or in their motivation to do so (Botvinick \& Braver, 2015). While we don't focus on individual differences here, our experimental approach should be useful for exploring why emotional distractions are merely a nuisance for some, but can be debilitating for others. Emotional distraction has been implicated in a number of disorders 
including depression, anxiety, and addiction (Cisler \& Koster, 2010; De Raedt \& Koster, 2010; Field \& Cox, 2008), and has been interpreted as both a bottom-up attentional bias and a top-down failure of control. The paradigm we have described here, which yields robust emotional distraction under reactive conditions, but effective control under proactive conditions, should therefore be very useful for probing the mechanisms that drive emotional distraction in these pathological conditions.

By examining emotional distraction through the lens of cognitive control we find the answer to a real-life puzzle. Emotional stimuli are everywhere. If they always distracted us, how would we get anything done? The Dual Mechanisms of Control framework provides an elegant solution. Emotional stimuli are important, but they aren't "magic" (see Pourtois et al., 2013). Their ability to distract us depends on the flexible interplay between proactive mechanisms that allow us to focus on our goals, and reactive mechanisms that keep us sensitive to novel threats and rewards.

Author Note: We thank Megan Parry for assistance with data collection in Experiment 3. Research was supported by a grant from the Royal Society of New Zealand Marsden Fund (VUW1307) to Gina Grimshaw and David Carmel. Portions of this research were presented at the 2015 meeting of the European Conference on Vision, and at the 2017 meeting of the Australasian Society for Experimental Psychology (EPC). 


\section{References}

Aue, T., Chauvigné, L. A. S., Bristle, M., Okon-Singer, H., \& Guex, R. (2016). Expectancy influences on attention to threat are only weak and transient: Behavioral and physiological evidence. Biological Psychology, 121, 173-186. doi:10.1016/j.biopsycho.2016.07.006

Aue, T., Guex, R., Chauvigné, L. A. S., \& Okon-Singer, H. (2013). Varying expectancies and attention bias in phobic and non-phobic individuals. Frontiers in Human Neuroscience, 7:418. doi: 10.3389/fnhum.2013.00418

Augst, S., Kleinsorge, T., \& Kunde, W. (2014). Can we shield ourselves from task disturbance by emotion-laden stimulation? Cognitive, Affective, and Behavioral Neuroscience, 14, 1009-1025. doi: 10.3758/s13415-013-0243-x

Botvinick, M., \& Braver, T. (2015). Motivation and cognitive control. The Annual Review of Psychology, 66, 83-113. doi:10.1146/annurev-psych-010814-015044

Botvinick, M. M., Braver, T. S., Barch, D. M., Carter, C. S., \& Cohen, J. D. (2001). Conflict monitoring and cognitive control. Psychological Review, 108(3), 624-652. doi:10.1037//0033-295X.108.3.624

Bradley, M. M., Codispoti, M., Sabatinelli, D., \& Lang, P. J. (2001). Emotion and motivation II: Sex differences in picture processing. Emotion, 1(3), 300-319. doi: 10.1037/15283542.1.3.300

Braver, T. S. (2012). The variable nature of cognitive control: A dual-mechanisms framework. Trends in Cognitive Sciences, 16(2), 106-113. doi:10.1016/j.tics.2011.12.010

Braver, T. S., Gray, J. R., \& Burgess, G. C. (2007). Explaining the many varieties of working memory variation: Dual mechanisms of cognitive control. In A. R. A. Conway, C. 
Jarrold, M. J. Kane, A. Miyake \& J. N. Towse (Eds.), Variation in working memory (pp.76-106). Oxford: Oxford University Press.

Braver, T. S., Paxton, J. L., Locke, H. S., \& Barch, D. M. (2009). Flexible neural mechanisms of cognitive control within human prefrontal cortex. Proceedings of the National Academy of Sciences, 106(18), 7351-7356. doi:10.1073pnas.080818

Bugg, J. M. (2012). Dissociating levels of cognitive control: The case of Stroop interference. Current Directions in Psychological Science, 21(5), 302-309. doi:10.1177/0963721412453586

Bugg, J. M., \& Crump, M. J. C. (2012). In support of a distinction between voluntary and stimulus-driven control: A review of the literature on proportion congruent effects. Frontiers in Psychology, 3: 367. doi:10.3389/fpsyg.2012.00367

Carretié, L. (2014). Exogenous (automatic) attention to emotional stimuli: A review. Cognitive, Affective, \& Behavioral Neuroscience, 14(4), 1228-1258. doi:10.3758/s13415-0140270-2

Casillas, A. \& Clark, L. A. (2000). The Mini Mood and Anxiety Symptom Questionnaire (MiniMASQ). Poster presented at the $72 \mathrm{bd}$ Annual Meeting of the Midwestern Psychological Association, Chicago, IL

Chiew, K. S., \& Braver, T. S. (2011). Positive affect versus reward: Emotional and motivational influences on cognitive control. Frontiers in Psychology, 2:279. doi:10.3389/fpsyg.2011.00279

Chiew, K. S., \& Braver, T. S. (2013). Temporal dynamics of motivation-cognitive control interactions revealed by high-resolution pupillometry. Frontiers in Psychology, 4:15. doi:10.3389/fpsy.2013.00015 
Cisler, J. M., \& Koster, E. H. W. (2010). Mechanisms of attentional biases towards threat in the anxiety disorders: An integrative review. Clinical Psychology Review, 30(2), 203-216. doi:10.1016/j.cpr.2009.11.003

De Raedt, R., \& Koster, E. H. W. (2010). Understanding vulnerability for depression from a cognitive neuroscience perspective: A reappraisal of attentional factors and a new conceptual framework. Cognitive, Affective, \& Behavioral Neuroscience, 10(1), 50-70. doi:10.3758/CABN.10.1.50

Derryberry, D., \& Reed, M. A. (2002). Anxiety-related attentional biases and their regulation by attentional control. Journal of Abnormal Psychology, 111(2), 225-236. doi: 10.1037/0021-843X.111.2.225

Desimone, R., \& Duncan, J. (1995). Neural mechanisms of selective visual attention. Annual Review of Neuroscience, 18(1), 193-222. doi:10.1146/annurev.ne.18.030195.001205

Devue, C., Bepolsky, A.V., \& Theeuwes, J. (2011). The role of fear and expectancies in capture of covert attention by spiders. Emotion, 11(4), 768-775. doi: 0.1037/a0023418

Dishon-Berkovits, M., \& Algom, D. (2000). The Stroop effect: It is not the robust phenomenon that you have thought it to be. Memory and Cognition, 28(8), 1437-1449.

Dunlap, W. P., Cortina, J. M., Vaslow, J. B., \& Burke, M. J. (1996). Meta-analysis of experiments with matched groups or repeated measures designs. Psychological Methods, 1(2), 170-177. doi: 10.1037/1082-989X.1.2.170.

Egner, T., \& Hirsch, J. (2005). Cognitive control mechanisms resolve conflict through cortical amplification of task-relevant information. Nature Neuroscience, 8(12), 1784-1790. doi:10.1038/nn1594

Field, M., \& Cox, W. M. (2008). Attentional bias in addictive behaviors: A review of its development, causes, and consequences. Drug and Alcohol Dependence, 97(1-2), 1- 
20. doi:10.1016/j.drugalcdep.2008.03.030

Folk, C. L., Remington, R. W., \& Johnston, J. C. (1992). Involuntary covert orienting is contingent on attentional control settings. Journal of Experimental Psychology: Human Perception and Performance, 18(4), 1030-1044. doi:10.1037/0096-1523.18.4.1030

Forster, S., \& Lavie, N. (2008a). Attentional capture by entirely irrelevant distractors. Visual Cognition, 16(2-3), 200-214. doi:10.1080/13506280701465049

Forster, S., \& Lavie, N. (2008b). Failures to ignore entirely irrelevant distractors: The role of load. Journal of Experimental Psychology: Applied, 14(1), 73-83. doi:10.1037/1076898X.14.1.73

Forster, S., \& Lavie, N. (2016). Establishing the attention-distractibility trait. Psychological Science, 27(2), 203-212. doi:10.1177/0956797615617761

Fox, E., Dutton, K., Yates, A., Georgiou, G. A., \& Mouchlianitis, E. (2015). Attentional control and suppressing negative thought intrusions in pathological worry. Clinical Psychological Science, 3(4), 593-606. doi:10.1177/2167702615575878

Fox, E., Russo, R., \& Dutton, K. (2002). Attentional bias for threat : Evidence for delayed disengagement from emotional faces. Cognition and Emotion, 16(3), 355-379. doi : $10.1080 / 02699930143000527$

Fox, E., Yates, A., \& Ashwin, C. (2012). Trait anxiety and perceptual load as determinants of emotion processing in a fear conditioning paradigm. Emotion, 12(2), 236-249. doi:10.1037/a0025321

Foxe, J. J., \& Snyder, A. C. (2011). The role of alpha-band brain oscillations as a sensory suppression mechanism during selective attention. Frontiers in Psychology, 2:154. doi:10.3389/fpsyg.2011.00154 
Gaspar, J. M., \& McDonald, J. J. (2014). Suppression of salient objects prevents distraction in visual search. The Journal of Neuroscience, 34(16), 5658-5666. doi:10.1523/JNEUROSCI.4161-13.2014

Gaspelin, N., Leonard, C. J., \& Luck, S. J. (2017). Suppression of overt attentional capture by salient-but-irrelevant color singletons. Attention, Perception, and Psychophysics, 79, 45-62. doi: 10.3758/s13414-016-1209-1

Gaspelin, N., Leonard, C. J., \& Luck, S. J. (2015). Direct evidence for active suppression of salient-but-irrelevant sensory inputs. Psychological Science, 26(11), 1740-1750. doi:10.1177/0956797615597913

Geng, J. J. (2014). Attentional mechanisms of distractor suppression. Current Directions in Psychological Science, 23(2), 147-153. doi:10.117/0963721414525780

Geyer, T., Müller, H. J., \& Krummenacher, J. (2008). Expectancies modulate attentional capture by salient color singletons. Vision Research, 48(11), 1315-1326. doi:10.1016/j.visres.2008.02.006

Gonthier, C., Braver, T. S., \& Bugg, J. M. (2016). Dissociating proactive and reactive control in the Stroop task. Memory and Cognition, 44, 778-788. doi: 10.3758/s13421-016-0591-1

Gupta, R., Hur, Y. J., \& Lavie, N. (2015). Distracted by pleasure: Effects of positive versus negative valence on emotional capture under load. Emotion. 16(3), 328-337. doi:10/1037/emo0000112

Hickey, C., Di Lollo, V., \& McDonald, J. J. (2009). Electrophysiological indices of target and distractor processing in visual search. Journal of Cognitive Neuroscience, 21(4), 760775. doi:10.1162/jocn.2009.21039 
Hilimire, M. R., Mounts, J. R., Parks, N. A., \& Corballis, P. M. (2011). Dynamics of target and distractor processing in visual search: Evidence from event-related brain potentials. Neuroscience Letters, 495(3), 196-200. doi:10.1016/j.neulet.2011.03.064

Kinsey, A. C., Pomeroy, W. R., \& Martin, C. E. (1948). Sexual behavior in the human male. Philadelphia PA: Saunders.

Lang, P. J., Bradley, M. M., \& Cuthbert, B. N. (2008). International affective picture system (IAPS): Affective ratings of pictures and instruction manual. Technical Report A-8. Gainesville, FL: University of Florida.

Li, F. F., VanRullen, R., Koch, C., \& Perona, P. (2002). Rapid natural scene categorization in the near absence of attention. Proceedings of the National Academy of Sciences, 99(14), 9596-9601. doi:10.1073/pnas.092277599

Lichtenstein-Vidne, L., Henik, A., \& Safadi, Z. (2012). Task relevance modulates processing of distracting emotional stimuli. Cognition and Emotion, 26(1), 42-52. doi: 10.1080/026999931.2011.567055

Maddock, A. T., Harper, D. N., Carmel, D., \& Grimshaw, G. M. (submitted). Motivation enhances control of positive and negative emotional distraction.

Mazaheri, A., DiQuattro, N. E., Bengson, J., \& Geng, J. J. (2011). Pre-stimulus activity predicts the winner of top-down vs. bottom-up attentional selection. PLoS One 6(2): e16243. doi: 10.1371/journal.pone.0016243

Mogg, K., Bradley, B. P., de Bono, J., \& Painter, M. (1997). Time course of attentional bias for threat information in non-clinical anxiety. Behavior Research and Therapy, 35(4), $297-$ 303. 
Most, S. B., Chun, M. M., Widders, D. M., \& Zald, D.H. (2005). Attentional rubber-necking: Cognitive control and personality in emotion-induced blindness. Psychonomic Bulletin and Review, 12(4), 654-661.

Most, S. B., Smith, S. D., Cooter, A. B., Levy, B. N., \& Zald, D. H. (2007). The naked truth: Positive, arousing distractors impair rapid target perception. Cognition and Emotion, 21(5), 964-981. doi:10.1080/02699930600959340

Morey, R. D. (2008). Confidence intervals from normalized data: A correction to Cousineau (2005). Tutorial in Quantitative Methods for Psychology, 4(2), 61-64.

Müller, H. J., Geyer, T., Zehetleitner, M., \& Krummenacher, J. (2009). Attentional capture by salient color singleton distractors is modulated by top-down dimensional set. Journal of Experimental Psychology: Human Perception and Performance, 35(1), 1-16. doi:10.1037/0096-1523.35.1.1

Neo, G., \& Chua, F. K. (2006). Capturing focused attention. Perception \& Psychophysics, 68(8), 1286-1296. doi:10.3758/BF03193728

Öhman, A., Flykt, A., \& Esteves, F. (2001). Emotion drives attention: Detecting the snake in the grass. Journal of Experimental Psychology: General, 130(3), 466-478. doi: 10.1037//0096-3445.130.3.466

Öhman, A., \& Mineka, S. (2001). Fears, phobias, and preparedness: Toward an evolved module of fear and fear learning. Psychological Review, 108(3), 483-522. doi: 10.1037//0033-295X.108.3.483

Okon-Singer, H., Lichtenstein-Vidne, L., \& Cohen, N. (2013). Dynamic modulation of emotional processing. Biological Psychology, 92(3), 480-491. doi:10.1016/j.biopsycho.2012.05.010 
Okon-Singer, H., Tzelgov, J., \& Henik, A. (2007). Distinguishing between automaticity and attention in the processing of emotionally significant stimuli. Emotion, 7(1), 147-157. doi:10.1037/1528-3542.7.1.147

Pearson, D., Donkin, C., Tran, S. C., Most, S. B., \& Le Pelley, M. E. (2015). Cognitive control and counterproductive oculomotor capture by reward-related stimuli. Visual Cognition, 23(1-2), 41-66. doi: 10.1080/13506285.2014.994252

Peelen, M. V., Li. F. F., \& Kastner, S. (2009). Neural mechanisms of rapid natural scene categorization in human visual cortex. Nature, 460(7251), 94-97. doi:10.1038/nature08103

Pessoa, L. (2009). How do emotion and motivation direct executive control? Trends in Cognitive Science, 13(4), 160-166. Doi: 10.1016/j.tics.2009.01.006

Phaf, R. H., \& Kan, K-J. (2007). The automaticity of emotional Stroop: A meta-analysis. Journal of Behavior Therapy and Experimental Psychiatry, 38, 184-199. Doi:10.1016/j.jbtep.2006.10.008

Pourtois, G., Schettino, A., \& Vuilleumier, P. (2013). Brain mechanisms for emotional influences on perception and attention: What is magic and what is not. Biological Psychology, 92(3), 492-512. doi: 10.1016/j.biopsycho.2012.02.007

Psychology Software Tools, Inc. [E-Prime 2.0]. (2012). Retrieved from http://www.pstnet.com.

Sawaki, R., \& Luck, S.J. (2010). Capture versus suppression of attention by salient singletons: Electrophysiological evidence for an automatic attend-to-me signal. Attention, Perception, and Psychophysics, 72(6), 1455-1470. doi: 10.3758/APP.72.6.1455

Theeuwes, J. (2010). Top-down and bottom-up control of visual selection. Acta Psychologica, 135, 77-99. doi: 10.1016/j.actpsy.2010.02.006 
Viviani, R. (2013). Emotion regulation, attention to emotion, and the ventral attentional network. Frontiers in Human Neuroscience, 7:746. doi:10.3389/fnhum.2013.00746

Vogt, J., De Houwer, J., Koster, E. H. W., Van Damme, S., \& Crombez, G. (2008). Allocation of spatial attention to emotional stimuli depends upon arousal and not valence. Emotion, 8(6), 880-885. doi: 10.1037/a0013981

Vromen, J. M., Lipp, O. V., \& Remington, R. W. (2015). The spider does not always win the fight for attention: Disengagement from threat is modulated by goal set. Cognition and Emotion, 29(7), 1185 - 1196. doi: 10.1080/026999931.2104.969198

Willenbockel, V., Sadr, J., Fiset, D., Horne, G. O., Gosselin, F. \& Tanaka, J. W. (2010). Controlling low-level image properties: the SHINE toolbox. Behavior Research Methods, 42(3), 671-684. doi:10.3758/BRM.42.3.671

Williams, J. M. G., Mathews, A., \& MacLeod, C. (1996). The emotional stroop task and psychopathology. Psychological Bulletin, 120(1), 3-24.

Yates, A., Ashwin, C., \& Fox, E. (2010). Does emotion processing require attention? The effects of fear conditioning and perceptual load. Emotion, 10(6), 822-830. doi:10.1037/a0020325

Yiend, J. (2010). The effects of emotion on attention: A review of attentional processing of emotional information. Cognition and Emotion, 24(1), 3-47. doi:10.1080/0269993090305698 
Table 1

Mean valence and arousal ratings (SD) for images presented to male and female participants

\begin{tabular}{lllllll}
\hline & & Valence & & & Arousal & \\
& Negative & Neutral & Positive & Negative & Neutral & Positive \\
\hline \multirow{2}{*}{ Men } & $1.83(.18)$ & $4.97(.31)$ & $7.09(.36)$ & $6.43(.41)$ & $3.15(.42)$ & $6.39(.51)$ \\
Women & $1.64(.21)$ & $5.01(.34)$ & $6.23(.52)$ & $6.53(.42)$ & $3.07(.32)$ & $6.31(.29)$ \\
\hline
\end{tabular}

Note. Ratings taken from published norms for men and women (Lang et al., 2008). Valence scaled from 1 (pleasant) to 9 (unpleasant); arousal scaled from 1 (low) to 9 (high).

IAPS image numbers used for female participants were: neutral $=2026,2102,2221,2305,2393$, 2397, 2411, 2512, 2593, 2595, 2745.1, 2840; negative = 3015, 3030, 3059, 3103, 3131, 3140, 3150, 3159, 3550.1, 9253, 9405, 9420; positive $=4658,4659,4660,4668,4680,4690,4693,4694,4695$, $4697,4698,4800$.

IAPS image numbers used for male participants were: neutral =2026, 2102, 2104, 2221, 2393, 2397, 2411, 2512, 2593, 2595, 2475.1, 2840; negative = 3000, 3015, 3053, 3060, 3069, 3071, 3080, 3100, 3120, 3130, 3131, 9410; positive $=4645,4650,4653,4658,4660,4666,4669,4680,4690,4692$, 4693,4698 
Table 2

Mean RT and Distraction Indices (with SDs) for Experiment 1, in ms

\begin{tabular}{rccccccc}
\hline \multicolumn{1}{l}{ Condition } & $\begin{array}{c}\text { Distractor } \\
\text { Present }\end{array}$ & $\begin{array}{c}\text { Distractor } \\
\text { Absent }\end{array}$ & $\begin{array}{c}\text { Distraction } \\
\text { Index }\end{array}$ & & & \multicolumn{2}{c}{$95 \% \mathrm{Cl}$} \\
\hline Low Frequency $(\mathrm{N}=48)$ & & & & & & & \\
Lower & Upper \\
Negative & $657(165)$ & $588(95)$ & $69(95)$ & $5.04^{* * *}$ & .371 & 41 & 96 \\
Neutral & $609(106)$ & $585(90)$ & $24(36)$ & $4.80^{* * *}$ & .228 & 14 & 35 \\
Positive & $651(145)$ & $581(89)$ & $70(80)$ & $6.04^{* * *}$ & .438 & 47 & 93
\end{tabular}

High Frequency $(\mathrm{N}=48)$

$\begin{array}{cccccccc}\text { Negative } & 581(101) & 568(93) & 13(32) & 2.86^{* *} & .131 & 4 & 22 \\ \text { Neutral } & 576(91) & 578(85) & -2(28) & 0.45 & .02 & -10 & 6 \\ \text { Positive } & 581(105) & 569(90) & 12(36) & 2.31^{*} & .112 & 2 & 23\end{array}$

Note: Distraction index $=[R T$ (distractor present) $-R T$ (distractor absent)]. $t$-values and effect sizes are from paired comparisons of distractor-present to distractor-absent trials, and indicate whether images caused significant distraction in that condition. 95\% confidence intervals surround the distraction index, in ms

${ }^{*} p<.05,{ }^{* *} p<.01, * * * p<.001$ 
Table 3

Mean Percent Accuracy and Distraction Indices (with SDs) for Experiment 1

\begin{tabular}{rcccccccc}
\hline \multicolumn{1}{l}{ Condition } & $\begin{array}{c}\text { Distractor } \\
\text { Present }\end{array}$ & $\begin{array}{c}\text { Distractor } \\
\text { Absent }\end{array}$ & $\begin{array}{c}\text { Distraction } \\
\text { Index }\end{array}$ & & & \multicolumn{2}{c}{$95 \% \mathrm{Cl}$} \\
\hline Low Frequency $(\mathrm{N}=48)$ & & & & $d_{r m}$ & Lower Upper \\
Negative & $96.18(5.21)$ & $96.64(2.54)$ & $0.46(5.04)$ & 0.64 & .108 & -1.00 & 1.93 \\
Neutral & $96.57(2.74)$ & $96.11(3.04)$ & $-0.46(3.01)$ & 1.07 & .160 & -1.34 & 0.41 \\
Positive & $94.79(5.86)$ & $95.89(3.30)$ & $1.10(5.31)$ & 1.44 & .219 & -0.44 & 2.64
\end{tabular}

High Frequency $(\mathrm{N}=48)$

$\begin{array}{cccccccc}\text { Negative } & 96.12(3.00) & 96.09(4.97) & -0.03(4.59) & -0.04 & .007 & -1.36 & 1.30 \\ \text { Neutral } & 95.93(2.95) & 96.66(3.24) & 0.72(3.86) & 1.30 & .233 & -0.40 & 1.84 \\ \text { Positive } & 96.41(3.50) & 96.87(3.70) & 0.46(4.66) & 0.69 & .129 & -0.89 & 1.82\end{array}$

Note: Distraction index = [accuracy (distractor absent) - accuracy (distractor present)]. $t$-values and effect sizes are from paired comparisons of distractor-present to distractor-absent trials, and indicate whether images caused significant distraction in that condition. 95\% confidence intervals surround the distraction index, in $\mathrm{ms}$

${ }^{*} p<.05, * * p<.01, * * * p<.001$ 
Table 4

Mean RT and Distraction Indices (with SDs) for Experiment 2, in ms

\begin{tabular}{|c|c|c|c|c|c|c|c|c|}
\hline \multirow[t]{2}{*}{ Condition } & & \multirow{2}{*}{$\begin{array}{c}\text { Distractor } \\
\text { Present }\end{array}$} & \multirow{2}{*}{$\begin{array}{c}\text { Distractor } \\
\text { Absent }\end{array}$} & \multirow{2}{*}{$\begin{array}{l}\text { Distraction } \\
\text { Index }\end{array}$} & \multicolumn{4}{|c|}{$95 \% \mathrm{Cl}$} \\
\hline & & & & & $t$ & $d_{r m}$ & Lower & Upper \\
\hline \multicolumn{9}{|c|}{ Low Frequency $(\mathrm{N}=40)$} \\
\hline & Negative & $610(97)$ & $564(73)$ & $46(51)$ & $5.69 * * *$ & .488 & 30 & 62 \\
\hline & Neutral & $594(99)$ & $571(80)$ & $23(36)$ & $4.00 * * *$ & .217 & 11 & 34 \\
\hline & Positive & $613(132)$ & $568(81)$ & $45(66)$ & $4.26 * * *$ & .273 & 23 & 65 \\
\hline
\end{tabular}

High Frequency $(\mathrm{N}=41)$

$\begin{array}{cccccccc}\text { Negative } & 586(100) & 575(95) & 11(49) & 1.48 & .115 & -4 & 27 \\ \text { Neutral } & 577(82) & 570(81) & 7(32) & 1.42 & .087 & -3 & 17 \\ \text { Positive } & 580(84) & 571(80) & 9(28) & 1.94 & .104 & 0 & 18\end{array}$

Combined Intact $(\mathrm{N}=42)$

$\begin{array}{cccccccc}\text { Negative } & 606(116) & 577(80) & 29(61) & 3.14^{* *} & .246 & 10 & 48 \\ \text { Neutral } & 586(90) & 567(77) & 19(38) & 3.30^{* *} & .218 & 7 & 31 \\ \text { Positive } & 599(117) & 569(92) & 30(49) & 4.02^{* * *} & .250 & 15 & 45\end{array}$

Combined Scrambled $(\mathrm{N}=42)$

$\begin{array}{cccccccc}\text { Negative } & 577(90) & 577(80) & 0(39) & 0.04 & .003 & -12 & 12 \\ \text { Neutral } & 568(76) & 567(77) & 1(28) & 0.39 & .022 & -7 & 10 \\ \text { Positive } & 571(97) & 569(92) & 2(33) & 0.31 & .017 & -9 & 12\end{array}$

Note: Distraction index $=[R T$ (distractor present) $-R T$ (distractor absent)]. $t$-values and effect sizes are from paired comparisons of distractor-present to distractor-absent trials, and indicate whether images caused significant distraction in that condition. 95\% confidence intervals surround the distraction index, in ms.

${ }^{*} p<.05, * * p<.01, * * * p<.001$ 
Table 5

Mean Accuracy (percent) and Distraction Indices (with SDs) for Experiment 2

\begin{tabular}{|c|c|c|c|c|c|c|c|c|}
\hline \multirow{2}{*}{\multicolumn{2}{|c|}{ Condition }} & \multirow{2}{*}{$\begin{array}{c}\text { Distractor } \\
\text { Present }\end{array}$} & \multirow{2}{*}{$\begin{array}{c}\text { Distractor } \\
\text { Absent }\end{array}$} & \multirow{2}{*}{$\begin{array}{l}\text { Distraction } \\
\text { Index }\end{array}$} & \multirow[t]{2}{*}{$\mathrm{t}$} & \multirow[t]{2}{*}{ d } & \multicolumn{2}{|c|}{$95 \% \mathrm{Cl}$} \\
\hline & & & & & & & Lower & Upper \\
\hline \multicolumn{9}{|c|}{ Low Frequency $(\mathrm{N}=40)$} \\
\hline & Negative & $96.04(4.71)$ & $96.46(3.27)$ & $0.42(3.96)$ & 0.67 & .099 & -0.85 & 1.68 \\
\hline & Neutral & 97.19 (3.70) & $96.56(3.27)$ & $-0.63(3.40)$ & -1.16 & .178 & -1.71 & 0.46 \\
\hline & Positive & $96.87(3.86)$ & 97.01 (2.89) & $0.14(3.36)$ & 0.26 & .040 & -0.93 & 1.21 \\
\hline
\end{tabular}

High Frequency $(\mathrm{N}=41)$

$\begin{array}{cccccccc}\text { Negative } & 96.75(4.17) & 97.56(3.36) & 0.81(4.38) & 1.19 & .213 & -0.57 & 2.20 \\ \text { Neutral } & 97.36(2.69) & 96.85(4.54) & -0.51(4.2) & -0.77 & .131 & -1.85 & 0.83 \\ \text { Positive } & 96.38(3.30) & 97.87(3.38) & 1.49(3.48) & 2.74^{* *} & .447 & 0.39 & 2.59\end{array}$

Combined Intact ( $\mathrm{N}=42$ )

$\begin{array}{llllllll}\text { Negative } & 96.13(5.24) & 97.42(3.56) & 1.29(5.33) & 1.57 & .283 & -0.37 & 2.95 \\ \text { Neutral } & 98.02(3.35) & 95.54(6.20) & -2.48(6.04) & 2.66^{*} & .480 & -4.36 & -0.60 \\ \text { Positive } & 96.53(5.03) & 96.035 .04) & -0.50(5.76) & -0.56 & .099 & -2.29 & 1.30\end{array}$

Combined Scrambled $(\mathrm{N}=42)$

$\begin{array}{cccccccc}\text { Negative } & 96.13(3.00) & 97.42(3.56) & 1.29(3.77) & 2.22^{*} & .390 & 0.12 & 2.46 \\ \text { Neutral } & 96.08(2.95) & 95.54(6.20) & -0.55(5.93) & -0.60 & .107 & -2.39 & 1.30 \\ \text { Positive } & 96.68(3.22) & 96.035 .04) & -0.65(4.97) & -0.84 & .149 & -2.19 & 0.90\end{array}$

Note: Distraction index $=$ [accuracy (distractor absent) - accuracy (distractor present)]. $t$-values and effect sizes are from paired comparisons of distractor-present to distractor-absent trials, and indicate whether images caused significant distraction in that condition. 95\% confidence intervals surround the distraction index, in ms.

${ }^{*} p<.05, * * p<.01$ 


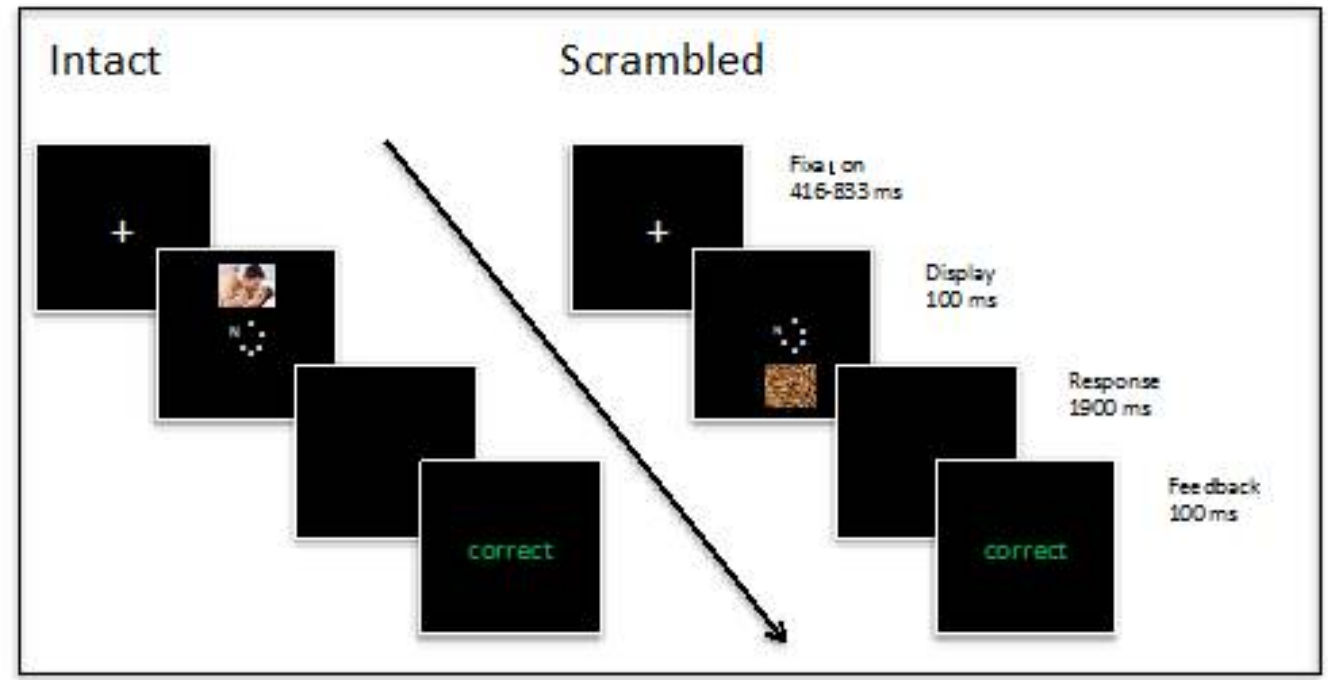

Figure 1. Trial procedure in both experiments. Distractors could appear either above or below the target display. Only intact images were presented in Experiment 1; both intact and scrambled images were presented in Experiment 2. Image shown is for display purposes; actual images were drawn from the International Affective Picture System (IAPS; Lang, Bradley, \& Cuthbert, 2008). Displays not to scale. 


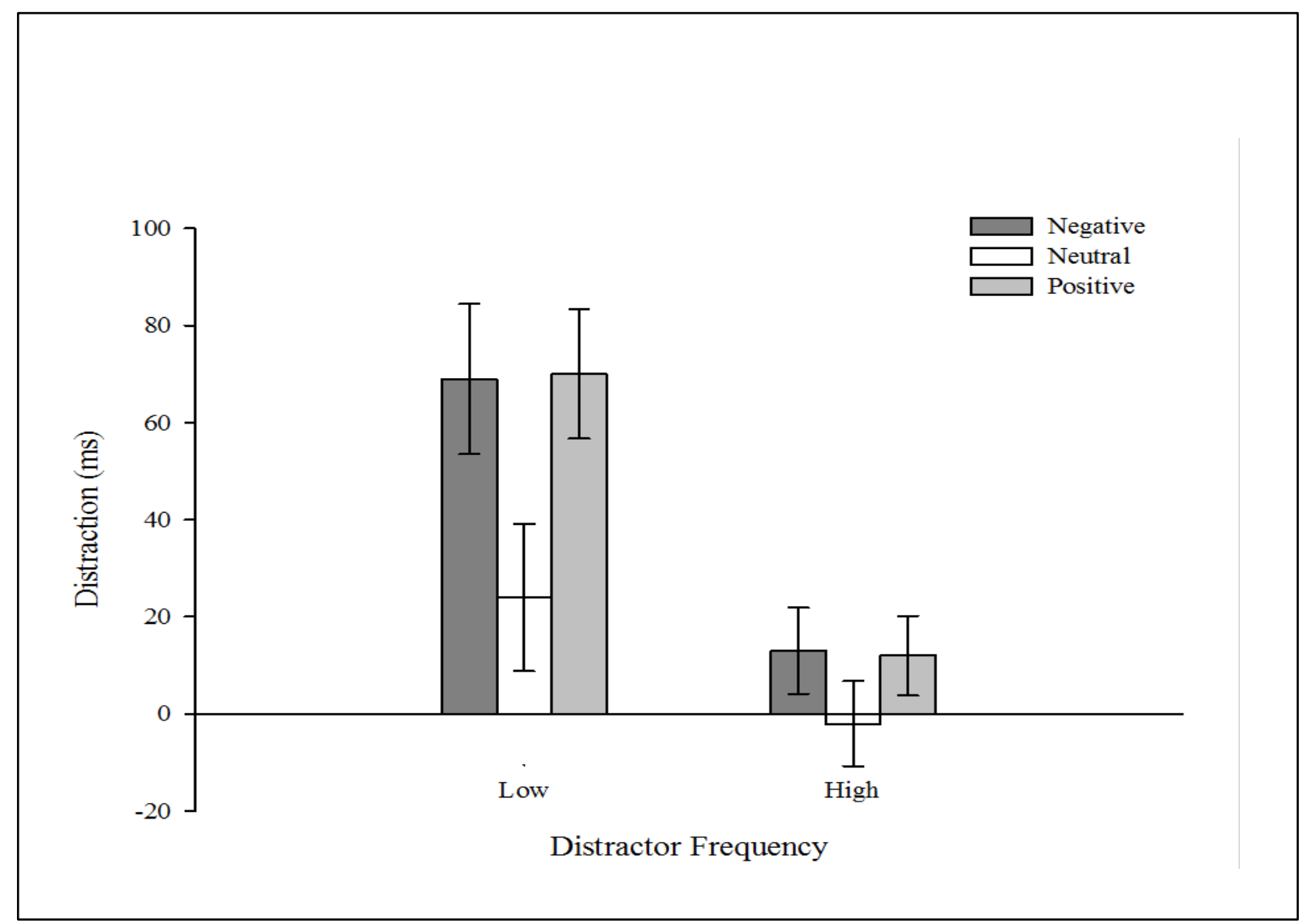

Figure 2. Distraction for participants in the low $(N=48)$ and high $(N=48)$ frequency conditions of Experiment 1. Error bars are 95\% confidence intervals for within-subject comparisons. 


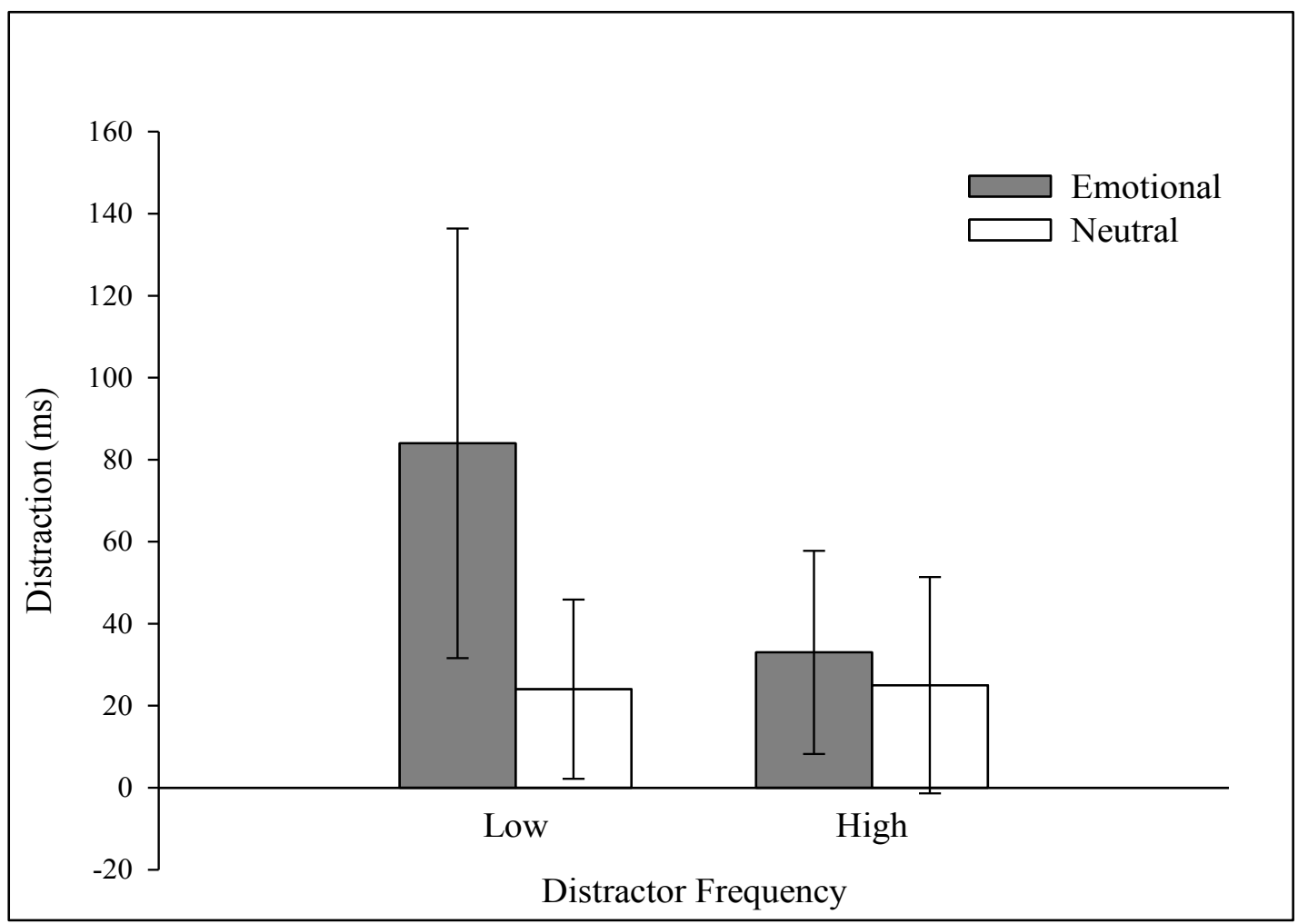

Figure 3. Distraction across initial trials of Experiment 1. Distraction was calculated using only the first 12 distractor-present and distractor-absent trials in the first block, and presented as a function of emotionality under high and low distractor frequency ( $N=24$ per condition). Error bars are $95 \%$ confidence intervals. 


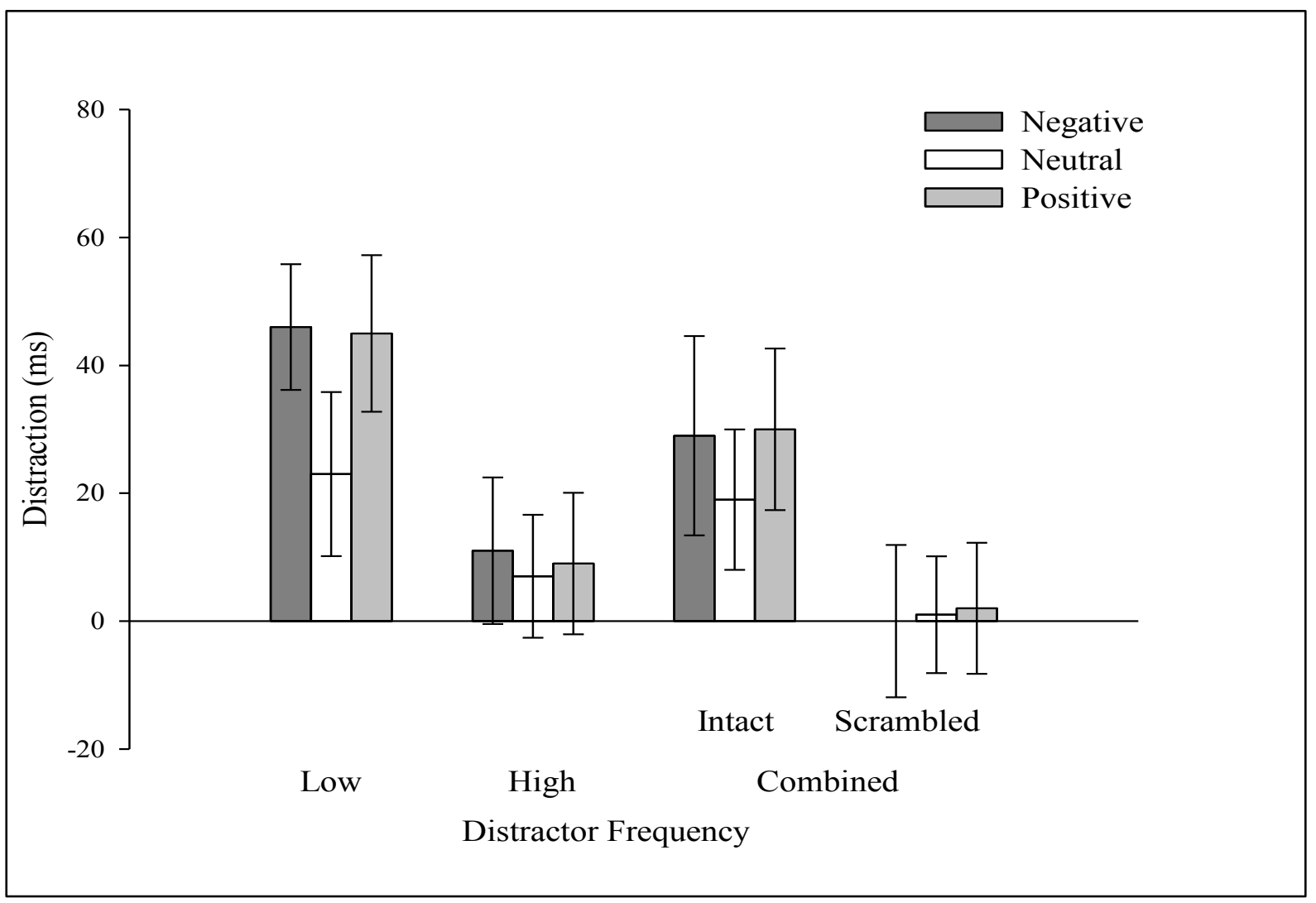

Figure 4. Distraction indices in Experiment 2. Results are shown for the low $(N=40)$, high $(N=41)$ and combined $(N=42)$ frequency conditions. In the combined condition, participants saw intact (25\%) and scrambled (50\%) distractors. Error bars are 95\% confidence intervals for within-subject comparisons. 